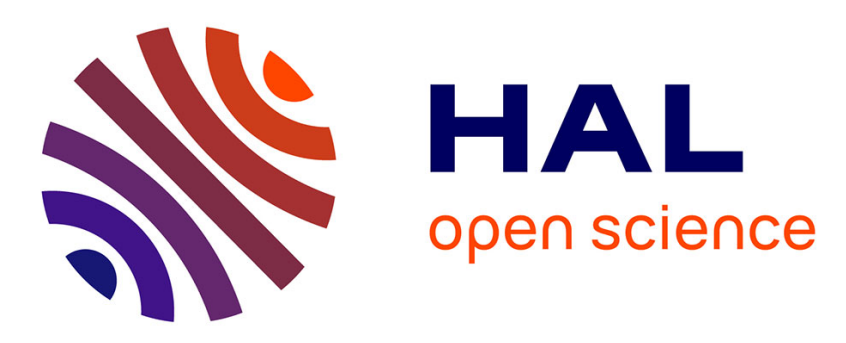

\title{
Some Modeling \& Simulation Perspectives on Emergence in System-of-Systems
}

\author{
Bernard P. Zeigler, Alexandre Muzy
}

\section{To cite this version:}

Bernard P. Zeigler, Alexandre Muzy. Some Modeling \& Simulation Perspectives on Emergence in System-of-Systems. Spring Simulation Multi-conference (SpringSim'16), Apr 2016, Pasadena, CA, United States. hal-01315199

\author{
HAL Id: hal-01315199 \\ https://hal.science/hal-01315199
}

Submitted on 17 May 2016

HAL is a multi-disciplinary open access archive for the deposit and dissemination of scientific research documents, whether they are published or not. The documents may come from teaching and research institutions in France or abroad, or from public or private research centers.
L'archive ouverte pluridisciplinaire HAL, est destinée au dépôt et à la diffusion de documents scientifiques de niveau recherche, publiés ou non, émanant des établissements d'enseignement et de recherche français ou étrangers, des laboratoires publics ou privés. 


\section{Some Modeling \& Simulation Perspectives on Emergence in System-of-Systems}

\author{
Bernard P. Zeigler \\ Chief Scientist, RTSync Corp \\ 12500 Park Potomac Ave. \\ Potomac, MD \\ United-States of America \\ zeigler@rtsync.com
}

\author{
Alexandre Muzy \\ I3S UMR CNRS 7271 \\ 06903 Sophia-Antipolis Cedex \\ France \\ alexandre.muzy@cnrs.fr
}

\begin{abstract}
We consider the ability of Discrete Event System Specification (DEVS) to provide the concepts and formalisms needed for modeling and simulation of emergent behavior. We show that DEVS provides systems components and coupling for models of systems of systems with emergent behavior. Further, DEVS coupling supports dynamic structure for adaptive and evolution, and the Experimental Frame supports Emergence Behavior Observation, and a recent extension, DEVS Markov models, supports prediction of emergence derived from such observation. Finally, we introduce a concept of interactive specification based on generators that has the potential to provide a system-theoretic characterization of emergence modeling using language concepts.
\end{abstract}

\section{INTRODUCTION}

Emergence as it has been recently treated has both subjective and objective aspects. Objectively for emergence to be observed, there are changes in the system that surprise the observer. However, such changes may not be significant enough to cause a more fundamental shakeup in understanding. Mittal [5] makes the point that "strong" emergent behavior results in generation of new knowledge about the system in the form of one or more of new abstraction levels and linguistic descriptions, new hierarchical structures and couplings, new component behaviors, and new feedback loops representing previously unperceived complex interactions. Once understood and curated, the behavior returns to the weak form, as it is no longer intriguing, and then can begin to be treated in regularized fashion. Moreover, emergent behavior is likely an inherent feature of any complex system model because abstracting a continuous real-world system (e.g. any complex natural system) to a constructed system-model must leave gaps of representation that may diverge in unanticipated directions. Since abstraction is needed to limit the inherently infinite statespace to a finite set of tractable and semantically labelled states, Mittal [7] argues that the model's dynam- ical behavior must account for the elapsed continuous time in the interval between any pair of successive states. This is the case for any computational model whether nominally continuous or discrete. Moreover, high performance computing and big data allow more points to fill the gaps but can never cover the space completely (cf. the mathematics of rationals and reals). Philosophically, following Ashby [1] and Foo and Zeigler [3], the perceived global behavior (holism) of a model might be characterized as: components (reductionism) + interactions (computation) + higher-order effects where the latter can be considered as the source of emergent behaviors $[4,12]$. In this paper, we present some features of DEVS that make it the right formalism to use to support the abstraction and observation necessary to deal with emergence in complex systems. We will make the following points:

- DEVS provides the components and couplings for models of complex systems

- DEVS supports dynamic structure for genuine adaption and evolution

- Experimental Frame supports Emergence Behavior Observation

- DEVS Markov models supports prediction of emergence

- DEVS enables fundamental emergence modeling

In the rest of this short paper, we briefly discuss these points.

\section{DEVS PROVIDES THE COMPONENTS AND COU- PLING FOR MODELS OF COMPLEX SYSTEMS}

Components and couplings in complex system models must include representation of decision making, in natural and artificial environments. DEVS has the universality [14] to represent the discrete (for agent models) and continuous (for natural environments) as well as hybrid (for artificial environments) formalism types needed for adequate complex system model construction. DEVS supports dynamic structure for genuine adaption and evolution. Strong dynamic structure capabilities are needed to specify and flexibly control the changes in 
components and their coupling to be able to adequately model adaptation, evolution and emergence in ways that include the possibility of genuine surprise. A next generation of dynamic structure formalisms have been recently under development in the DEVS community $[9,11,10]$. We briefly review the concepts here. First an overall framework capable of representing existing formulations introduced by [8] is shown below framed in terms of iterative specifications [14]. Such a specification is based on trajectory segments that together generate the complete set of input segments by composition. The basic idea is that a change in structure is a replacement of one DEVS model by another one with the latter starting from a state related to the last state of the former. The definition first deals with a basic model and goes on to the coupled model case.

\subsection{Basic dynamic structure}

Definition 1. Basic or atomic Dynamic Structure System Specification (DYS-SYS) structure

$$
D Y S-S Y S=(\mathcal{M}, \mathcal{Q}, \tau)
$$

Where each element $M \in \mathcal{M}$ is an iterative system specification $I O S G=\left(T, X, \Omega_{G_{X}}, Y, Q, \delta, \lambda\right)$ where $T$ is the time base, $X$ is the set of input values, $\Omega_{G_{X}}$ is the admissible set of input segment generators, $Y$ is the set of output values, $Q$ is the set of states, $\delta: Q \times \Omega_{G_{X}} \rightarrow$ $Q$ is the single segment state transition function, and $\lambda: Q \rightarrow Y$ is the output function; $\mathcal{Q}=\amalg_{M \in \mathcal{M}} Q_{M}$ is the disjoint union of the state sets of the models; and $\tau: \mathcal{M} \times \mathcal{S} \rightarrow \mathcal{M} \times \mathcal{S}$ is the structure transition function. Structure function $\tau$ takes a basic system IOSG and its state to a new basic IOSG' and a new state: $\tau(M, q)=\left(M^{\prime}, q^{\prime}\right)$. This represents a basic change in structure which transforms a basic DEVS into a new basic IOSG', by changing its structure in some way (one or many elements of $\left(T, X, \Omega_{G_{X}}, Y, Q, \delta, \lambda\right)$ and initializing the state of the new system.

\subsection{Dynamic structure network}

Definition 2. Dynamic Structure System Network structure

$$
D Y S-S Y N=(\mathcal{N}, Q, \tau)
$$

Where $\mathcal{N}=\left\{\left(X, Y, D,\left\{M_{d}\right\},\left\{I_{d}\right\},\left\{Z_{i, d}\right\}\right)\right\}$ is the set of network structures, where each component $d \in D$ is a an atomic dynamic structure model $M_{d}=\left(\mathcal{M}_{d}, \mathcal{Q}_{d}, \tau_{d}\right), \quad \mathcal{Q}_{d}=\amalg_{N \in \mathcal{N}} Q_{N}$ is the disjoint union of state sets of network structures, with $Q_{N}=\Pi_{d \in D} Q_{d}$ the partial state of a network $N \in \mathcal{N}$ is the crossproduct of the partial state sets of its components, and $\tau: \mathcal{N} \times \mathcal{Q} \rightarrow \mathcal{N} \times \mathcal{Q}$ is the structure transition function of the network.

Muzy and Zeigler [8] showed that this is a well-defined definition that preserves the DEVS closure under coupling and includes existing formulations [2, 13]. The motivation for the generalization was to free up the control of structure change from a single fixed point to allow multiple sources that might be active in a distributed simulation. In the example they give, a coupling is a static relationship between components that relies on collaboration between model components to achieve dynamic structural and connectivity permutation of those couplings. This is a kind of a client-server or a peer-topeer message communication/synchronization method. In contrast, Park [10] defined a universal coupling specification (UCS) which is different in that a coupling is a dynamic component that can perform permutation by itself based on its own constraints and requirements. In this approach, component models do not need to provide any particular logic to handle coupling changes. This allows separation of the dynamic structural and coupling permutation logic from the model behaviors unrelated to coupling.

In [10], Park provides a number of types of coupling restructurings inspired by micro-biological systems modeling that are potentially applicable to general modeling of emergence. In [11], Steiniger defines a concept of intensional coupling specification distinct from the explicit (extensional) coupling they specify. Biological cellular level modeling similarly motivated this development with the same implications for emergence modeling.

\section{EXPERIMENTAL FRAME SUPPORTS EMERGENCE BEHAVIOR OBSERVATION}

Mittal and Rainey [6] define Emergence Behavior Observers (EBO) that can observe system state transitions and are tuned to catch segments potential of interest and recording snapshots into memory. A snapshot is defined as an information-set comprising of components, their states, taken at specified moments for specified durations. The concept of Experimental Frame (EF) is a general concept which separates the model from the conditions under which it is observed and executed [14] and can include the more specific EBO concept.

\section{DEVS MARKOV MODELS SUPPORTS PREDICTION OF EMERGENCE}

DEVS Markov models support prediction of emergence Time series of snapshots can be compiled into DEVS Markov models [15] that offer more explanatory and predictive power than the raw inputs. Such compilation can proceed in real-time giving human observers of the simulation a heads-up on imminent events. Figure 1 illustrates the role of EBOs and Markov models in real-time emergence monitoring.

We start with a coupled model whose components are conceptually partitioned into blocks, each component's outputs are observed by an experimental frame, call the local EF. This frame samples its block's activity over a (moving) time interval by counting the changes in values of the outputs of the sampled components over the interval. The EF outputs an abstraction of the activity of the block at the end of each interval, e.g., as measured by the scale: zero, low, medium, high based a series of increasing thresholds for the total activity. The vector of these abstracted local states form an abstraction of the 


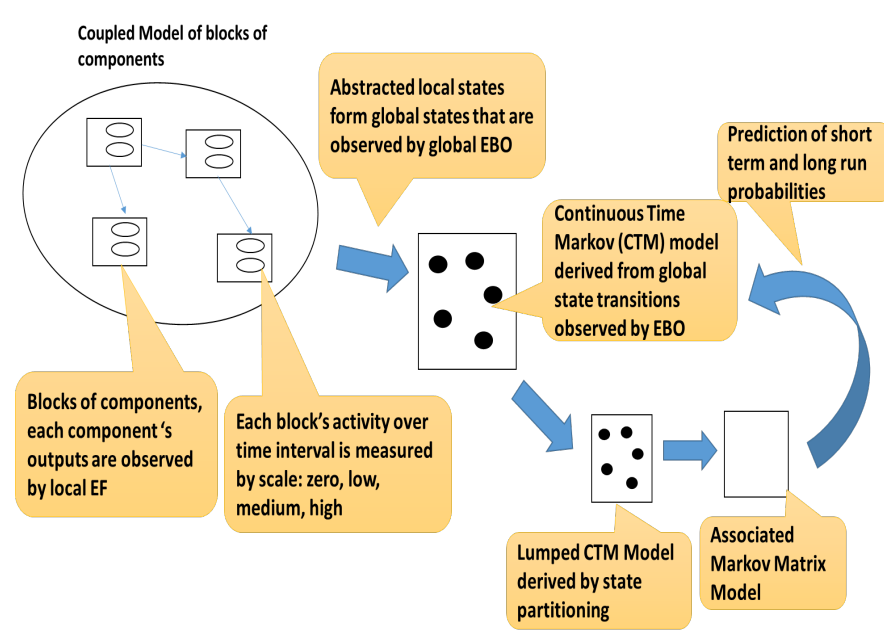

Figure 1. The role of Emergence Behavior Observation and Markov models in real-time emergence monitoring.

the global state of the system and is observed by global EBO. By compiling these state transitions, a Continuous Time Markov (CTM) model [15] is automatically derived. This model is a dynamic structure model as its structure is modifiable depending on the incoming sequence of state labels. Although more compact than the original system, this model may still be too large to comprehend. So it is possible to further reduce this model to a lumped CTM Model derived by state partitioning, the associated Markov chain matrix model can be employed online to provide predictions of short term and long run probability statistics. This enables human observers to understand the underlying cause of behavior deviations and predict when and where they might recur.

\section{DEVS ENABLES FUNDAMENTAL EMERGENCE MOD- ELING}

Emergence has been modeled at the fundamental level in terms of formation of new language elements from interaction of components whose behavior does not individually manifest such elements $[4,12]$. In the following, we show how the dynamic structure formalization given above can provide a mechanistic formulation of such emergence

\subsection{Formal language of dynamic systems}

An iterative system specification is characterized by a set of generator behaviors $\beta_{G}=$ $\left\{\left(\omega_{G_{X}}, \omega_{G_{Y}}\right) \mid\left(\omega_{G_{X}}, \omega_{G_{Y}}\right) \in \beta, \beta=X^{T} \times Y^{T}, \beta_{G} \subseteq\right.$ $\left.\beta, \operatorname{dom}\left(\omega_{G_{X}}\right)=\operatorname{dom}\left(\omega_{G_{Y}}\right)\right\}$. Each output segment generator $\omega_{G_{Y}} \in \Omega_{G_{Y}}$ can be considered as a word generated by an autonomous system over a time period equal to $\operatorname{dom}\left(\omega_{G_{Y}}\right)$.

\subsection{Emergence modeling}

Emergence can be observed as a difference between local and global levels of behaviors in the behavior set $\beta_{G}$ of an iterative I/O system specification $I O S G$. Imagine that you simulate a system at a first level and inductively collect all the behaviors for each state $q \in Q$ in $\beta_{G_{q \in Q}}$. Now, observing the behaviors at a higher level you also inductively collect all the behaviors for each new state $q^{\prime} \in Q^{\prime}$ in $\beta_{G_{q^{\prime} \in Q^{\prime}}^{\prime}}^{\prime}$. The mapping between both behavior levels can be achieved by a dynamic structure network called an emergence constructor $E=(\mathcal{N}, \mathcal{Q}, \tau)$, where $\beta_{G_{q \in Q}} \subset \beta_{G_{q^{\prime} \in Q^{\prime}}^{\prime}}, Q \subset Q^{\prime}$ for $\tau(N, Q)=\left(N^{\prime}, Q^{\prime}\right)$, and $N$ and $N^{\prime}$ structures only differing in component state set and transition definitions.

Example 1. Consider a network of spiking neurons. At first, we observe spikes being exchanged by the neurons. This can be formalized as a language $[4,12]$ in which the spikes are in a network generating $\beta_{G_{q \in Q}}$. Later we observe that the spikes tend to be grouped into clusters which can be formalized as bursts. These bursts can then be formalized as being in a network generating $\beta_{G_{q \in Q^{\prime}}}^{\prime}$.

\section{CONCLUSION AND PERSPECTIVE}

We have demonstrated the ability of DEVS to provide the concepts and formalisms needed for modeling and simulation of emergent behavior. DEVS has a number of features that enable the representation of systems components and couplings that can change dynamically as needed for genuine adaptation and evolution. We indicated how DEVS Markov models can support prediction of emergence derived from observation of complex system behavior. Finally, we introduced a concept of interactive specification based on generators that has the potential to provide a system-theoretic characterization of emergence modeling using language concepts. This concept can provide a way forward for more indepth understanding of emergence in systems of systems.

\section{REFERENCES}

1. Ashby, W. An Introduction to Cybernetics. University Paperbacks, Methuen, London, 1964.

2. Barros, F. J. Towards a theory of continuous flow models. International Journal of General Systems 31, 1 (2002), 29-40.

3. Foo, N. Y., and Zeigler, B. P. Emergence and computation. International Journal of General Systems 10, 2-3 (1985), 163-168.

4. Kubík, A. Toward a formalization of emergence. Artificial Life 9, 1 (2003), 41-65.

5. Mittal, S. Emergence in stigmergic and complex adaptive systems: A formal discrete event systems perspective. Cognitive Systems Research 21 (2013), 22 - 39. Stigmergy in the Human Domain.

6. Mittal, S., and Rainey, L. Harnessing emergence: The control and design of emergent behavior in system of systems engineering. In Proceedings of the 
Conference on Summer Computer Simulation, SummerSim '15, Society for Computer Simulation International (San Diego, CA, USA, 2015), 1-10.

7. Mittal, Saurabh, and Zeigler, Bernard P. Context and attention in activity-based intelligent systems. ITM Web of Conferences 3 (2014), 03001.

8. Muzy, A., and Zeigler, B. P. Specification of dynamic structure discrete event systems using single point encapsulated control functions.

International Journal of Modeling, Simulation, and Scientific Computing 05, 03 (2014), 1450012.

9. Ören, T. I., and Yilmaz, L. Awareness-based couplings of intelligent agents and other advanced coupling concepts for m\&amp;s. In SIMULTECH 2015 - Proceedings of the 5th International Conference on Simulation and Modeling Methodologies, Technologies and Applications, Colmar, Alsace, France, 21 - 23 July, 2015. (2015), 3-12.

10. Park, S., and Hunt, C. Coupling permutation and model migration based on dynamic and adaptive coupling mechanisms. In Agent Directed Simulation Conference/Spring Simulation Multiconference (Von Braun Center Huntsville, Alabama USA, 2006), 6-15.

11. Steiniger, A., and Uhrmacher, A. M. Intensional couplings in variable-structure models: An exploration based on multilevel-devs. ACM Trans. Model. Comput. Simul. 26, 2 (Jan. 2016), 9:1-9:27.

12. Szabo, C., and Teo, Y. M. Formalization of weak emergence in multiagent systems. ACM Trans. Model. Comput. Simul. 26, 1 (Sept. 2015), 6:1-6:25.

13. Uhrmacher, A. M. Dynamic structures in modeling and simulation: A reflective approach. ACM Trans. Model. Comput. Simul. 11, 2 (Apr. 2001), 206-232.

14. Zeigler, B., Praehofer, H., and Kim, T. Theory of Modeling and Simulation: Integrating Discrete Event and Continuous Complex Dynamic Systems. Academic Press, 2000.

15. Zeigler, B. P., Nutaro, J. J., and Seo, C. Combining devs and model-checking: Concepts and tools for integrating simulation and analysis. To appear in Int. J. Process Modeling and Simulation. Special Issue on: "New Advances in Simulation and Process Modelling: Integrating New Technologies and Methodologies to Enlarge Simulation Capabilities" (2016). 